\title{
Quantifying Strain Birefringence Haloes Around Inclusions in Diamond
}

\author{
Dan Howell $^{1}$, Adrian Jones ${ }^{1}$, David Dobson ${ }^{1}$, Ian Wood ${ }^{1}$, \\ Lutz Nasdala $^{2}$ \& Jeff Harris ${ }^{3}$ \\ ${ }^{1}$ Earth Sciences Department, University College London, UK \\ ${ }^{2}$ Institute of Mineralogy \& Crystallography, University of Vienna, Austria \\ ${ }^{3}$ Department of Geographical \& Earth Sciences, University of Glasgow, UK
}

Birefringence in diamond has long been recognized as an anomalous optical property for a cubic mineral (Lang, 1967). Various causes have been postulated for this phenomenon but they all identify birefringence as being the result of the photoelastic effect (change of refractive index with stress) (Poindexter, 1955). Until now birefringence studies (a non-destructive technique) have seldom been utilized because of the time consuming nature of the analysis. The MetriPol system is a new automated birefringence analysis system that can produce very accurate data in a matter of seconds (Glazer et al. 1996).

When analyzing stress-induced birefringence, it is important to consider the difference from normal birefringence. Whilst normal birefringence is the result of constant anisotropy throughout the sample, stressinduced birefringence is the result of anisotropy that is governed by the stress field. This means that a beam of light that passes through the radial stress field around an inclusion under remnant pressure will not record the peak value of anisotropy that it encounters but, in effect, a value that is an average over the entire thickness of the diamond. This means that it is important to understand the relationship between the inclusion size, diamond thickness, remnant pressure and recorded value of birefringence before any analysis can be interpreted with confidence. To quantify this relationship a theoretical model has been developed.

All studies into quantifying the elastic effects around inclusions in diamond that remain under remnant pressure are based upon a number of assumptions. In this study these assumptions are:

(i) The inclusion is spherical.

(ii) Both the host and inclusion have cubic crystal symmetry.

(iii) When formed, the diamond fits the inclusion perfectly with no interfacial region of different composition or structure.

(iv) Deformation is linear elastic and no brittle or plastic deformation has occurred.

(v) The remnant pressure of the inclusion is the only cause of strain in the diamond lattice that results in birefringence.

The model uses an inclusion (radius $=R_{\mathrm{i}}$ ) under remnant pressure $\left(P_{\mathrm{i}}\right)$ in the centre of a semi-infinite slab of diamond of thickness $L$. The remnant pressure creates a radial stress field in the host diamond that decays as $1 / r^{3}$. By splitting up a $2 \mathrm{D}$ section, that runs through the centre of the inclusion, into $s$ number of slices (Fig. 1), an optical indicatrix (related to the radial stress regime) can be calculated for a point in the centre of each slice. For a beam of light passing through the slab at a distance, $d$, from the centre of the inclusion, the retardation is calculated by summing the retardation from the indicatrix in each slice (1 to $s$ ). The peak value of retardation will be obtained when the light passes immediately next to the inclusiondiamond interface (when $d=R_{\mathrm{i}}$ ), and will decrease as it moves away from it $\left(d>R_{\mathrm{i}}\right)$.

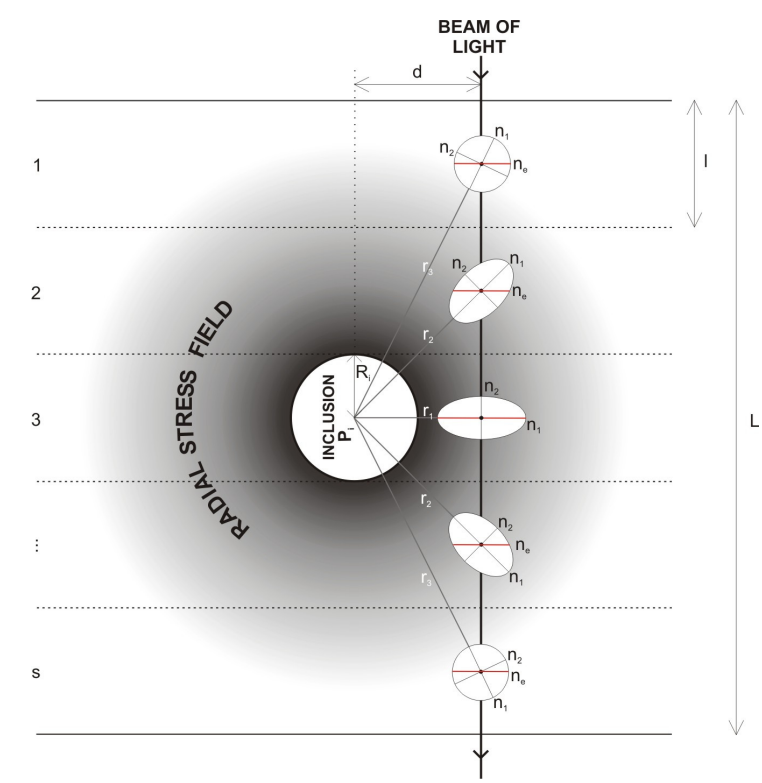

Fig. 1: schematic image explaining the parameters of the theoretical model.

It is clear that the measured birefringence value (through the radial stress regime) will be significantly less than a theoretical maximum birefringence value obtained from a stress that is equal to the remnant pressure of the inclusion. Using several different values for each of the 3 main variables $\left(R_{\mathrm{i}}, L \& P_{\mathrm{i}}\right)$, the model has been used to calculate the peak measured birefringence (when $d=R_{\mathrm{i}}$ ). This model has defined a relationship that involves all of these 4 variables, so that the remnant pressure on an inclusion can be estimated from the birefringence measurements. 
To test the validity of this relationship, a variety of analyses have been performed on diamonds with inclusions in. Techniques including X-ray analysis (Harris et al., 1970), 2D Raman mapping of the host (Nasdala et al., 2003; 2005), and Raman spot analysis of the inclusion (Liu et al., 1990, Izraeli et al., 1999) have already been documented for measuring remnant pressure on inclusions in diamond. So the results from these techniques have been used to compare with the results from MetriPol birefringence analysis system and the theoretical model.

There are a number of criteria that govern the use of diamond samples in this study. These are not only related to the assumptions already listed, but also due to the limitations of the MetriPol system and sample preparation capabilities. The birefringence analysis requires 2 parallel faces to view through. This means that natural diamonds with good octahedral faces can be utilized. However, it is rare to find samples in which the birefringence is only caused by an inclusion under remnant pressure, and not by plastic deformation, variations in nitrogen concentration, or dislocations (Lang, 1967). The diamond should also only have 1 inclusion in (to prevent any overlapping stress fields complicating the interpretation). Garnets are the preferred mineral inclusion due to their cubic crystal symmetry. This means that cubic minerals will apply an equal stress to the host diamond along each of its 3 main crystallographic axes.

From many collections of diamonds sampled, 3 natural octahedra containing garnet inclusions were chosen from Udachnaya, Siberia (sample numbers UD2, 5 \& 7). A prepared diamond plate containing 4 coesite inclusions from Finsch, South Africa (F125), has also been analyzed. Despite being monoclinic, coesite is interesting due to its calibrated pressure sensitive Raman peak (Hemley, 1987) and the resultant extraordinarily high levels of birefringence observed. The birefringence seen in these samples is shown in Figures 2, $3 \& 4$.

Using the MetriPol birefringence analysis and the model described above suggests that the garnet in UD7 is under $0.38 \pm 0.05 \mathrm{GPa}$ of remnant pressure. Without libera-ting the garnet inclusions from the host diamond, it is not possible to verify this result. The results from the liberated inclusions will be presented at the conference. This is not so simple to do for samples UD2 \& 5, as the birefringence patterns in them are far more complex. Figure 4 shows that the birefringence is not just focused around the inclusion but pervasive throughout the entire sample. In UD2 the birefringence pattern seems to be following the growth stratigraphy of the stone, while in UD5 the pattern suggests that it has been plastically deformed (near Type II diamond). For quantitative purposes, this highlights the importance of focusing on birefringence that is only caused by the inclusion. This makes finding suitable
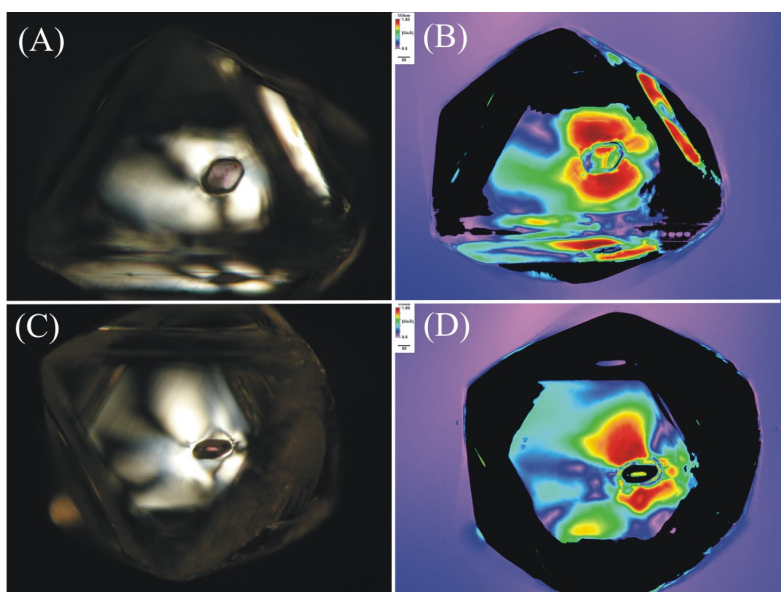

Fig. 2: Images of UD7 taken under crossed-polars (A $\& \mathrm{C})$ and the MetriPol system (B \& D).
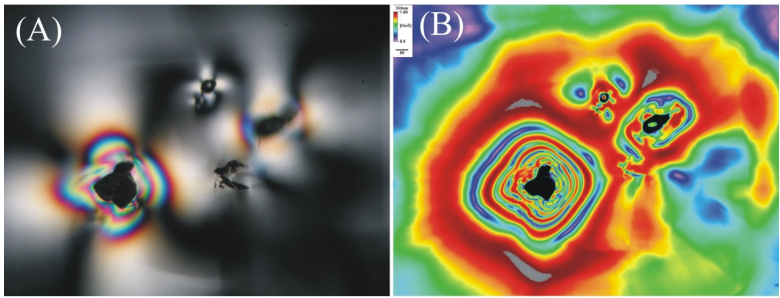

Fig. 3: Images of F125 taken under crossed-polars (A) and the MetriPol system (B).
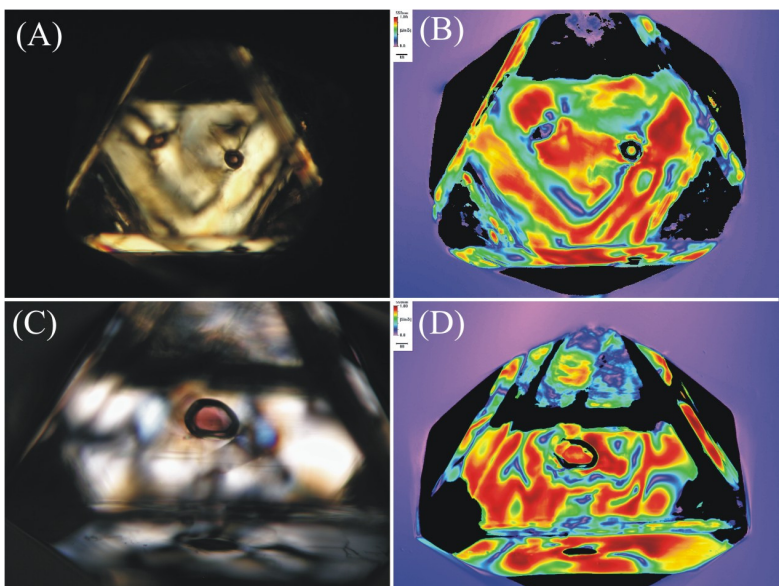

Fig. 4: Images of UD2 (A \& B) and UD5 (C \& D), show how growth zoning (UD2) and plastic deformation (UD5) can overwhelm the birefringence caused by remnant pressure on an inclusion.

samples very difficult, as the other causes of birefringence are quite common. For example, the entrapment of inclusions can result in dislocations in the host diamond due to lattice closure errors, fractures are commonly seen around inclusions, the diamond may have a complex growth stratigraphy caused by varying levels of nitrogen being incorporated into the lattice, and the sample may undergo plastic deformation due to non-hydrostatic stresses in the mantle. All of these possibilities need to be considered when interpreting the quantitative birefringence analysis with confidence. 
Applying the birefringence analysis and theory to the largest coesite inclusion in F125 gives a remnant pressure of $2.33 \pm 0.26 \mathrm{GPa}$. This is in agreement with the result from the Raman peak shift of $2.55 \pm 0.35$ GPa. This result suggests that as a first order approximation, the model can be applied to non-cubic mineral inclusions with good agreement. However, to be able to place a high level of confidence on the results of uniaxial and biaxial inclusions will require understanding of the effect of anisotropy on the radial stress field. Another important factor that may have an effect on the stress field is the shape of the inclusion. Finite element analysis calculations are currently being performed to assess these.

In conclusion, these preliminary results suggest that quantitative birefringence analysis with the MetriPol system is a very useful technique for assessing remnant pressure on inclusions in diamond. The rapid nature of the technique and the near isotropic nature of the effect suggest that this method may provide a comparable alternative to 2D Raman mapping. However, we are aware that many more analyses are required to support there results. This quantitative birefringence analysis is currently also being successfully used to investigate the other causes of birefringence in diamond to assess the stresses and strains that they produce.

\section{References}

Glazer, A.M., Lewis, J.G., Kaminsky, W., 1996. An automatic optical imaging system for birefringent media. Proceedings Royal Society London, 452, 2751-2765.

Harris, J.W., Milledge, H.J., Barron, T. Munn, R.W., 1970. Thermal expansion of garnets included in diamond. Journal of Geophysical Research, 75 (29) 5775-5792

Hemley, R.J., 1987. Pressure dependence of Raman spectra of $\mathrm{SiO}_{2}$ polymorphs: $\alpha$-quartz, coesite, and stishovite, in High-Pressure Research in Mineral Physics (eds. M.H. Manghnani and Y. Syono), pp. 347-359.

Izraeli, E.S., Harris, J.W., Navon, O., 1999. Raman barometry of diamond formation. Earth \& Planetary Science Letters, 173, 351-360.

Lang, A.R., 1967. Causes of birefringence in diamond. Nature, 213, 248-251.

Liu, L., Mernagh, T.P., Jaques, A.L., 1990. A mineralogical Raman spectroscopy study on eclogitic garnet inclusions in diamonds from Argyle. Contributions to Mineralogy \& Petrology, 105, 156-161.

Nasdala, L., Brenker, F.E., Glinnemann, J., Hofmeister, W., Gasparik, T., Harris, J.W., Stachel, T., Reese, I., 2003. Spectroscopic 2D-tomography: Residual pressure and strain around mineral inclusions in diamonds. European Journal of Mineralogy, 15, 931-935.

Nasdala, L., Hofmeister, W., Harris, J.W. Glinnemann, J., 2005. Growth zoning and strain patterns inside diamond crystal as revealed by Raman maps. American Mineralogist, 90, 745-748.

Poindexter, E., 1955. Piezobirefringence in diamond. American Mineralogist, 40, 1135-1139. 